\title{
Comparison of phenolic compositions between common and tartary buckwheat (Fagopyrum) sprouts.
}

\begin{abstract}
The phenolic compositions of non-germinated/germinated seeds and seed sprouts (at 6-10 day-old) of common (Fagopyrum esculentum Möench) and tartary (Fagopyrum tataricum Gaertn.) buckwheats were investigated. Phenolic compounds, including chlorogenic acid, four C-glycosylflavones (orientin, isoorientin vitexin, isovitexin), rutin and quercetin, were determined in the seed sprouts by high-performance liquid chromatography (HPLC). In the edible parts of common buckwheat sprouts, individual phenolics significantly increased during sprout growth from 6 to 10 days after sowing (DAS), whereas in tartary buckwheat sprouts they did not. While the sum contents of phenolic compounds in the edible part (mean $24.4 \mathrm{mg} / \mathrm{g}$ DW at 6-10 DAS) of tartary buckwheat sprouts were similar to those of common buckwheat sprouts, rutin contents in the non-germinated/germinated seeds (mean $14.7 \mathrm{mg} / \mathrm{g}$ DW) and edible parts (mean $21.8 \mathrm{mg} / \mathrm{g}$ DW) of tartary buckwheat were 49- and 5-fold, respectively, higher than those of common buckwheat. Extracts of the edible parts of both species showed very similar free radical-scavenging activities (mean $1.7 \mu$ mol trolox eq/g DW), suggesting that the overall antioxidative activity might be affected by the combination of identified phenolics and unidentified (minor) components. Therefore, buckwheat seed sprouts are recommended for their high antioxidative activity, as well as being an excellent dietary source of phenolic compounds, particularly tartary buckwheat sprouts, being rich in rutin.
\end{abstract}

Keyword: Buckwheat sprouts; DPPH assay; Functional food; Phenolic compounds and flavonoids. 